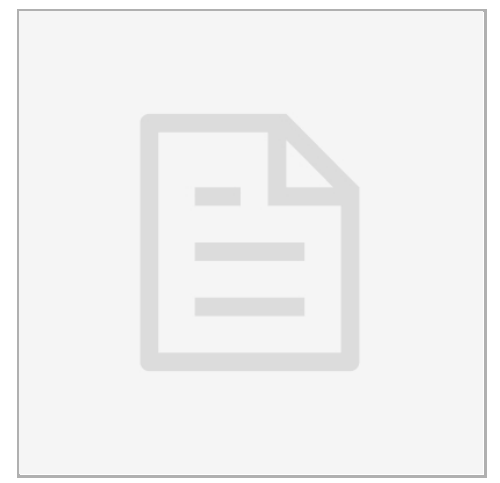

VERSION 1

APR 10, 2019

\section{open $\boldsymbol{O}$ access}

DOI:

dx.doi.org/10.17504/protocol s.io.zwxf7fn

\section{Protocol Citation: Amy} Lyden, Emily Crawford, Jenai Quan, Saharai Caldera, Lara Pesce-Ares 2019. In Vitro Transcription for dgRNA. protocols. io

https://dx.doi.org/10.17504/p rotocols.io.zwxf7fn

License: This is an open access protocol distributed under the terms of the Creative Commons Attribution License, which permits unrestricted use, distribution, and reproduction in any medium, provided the original author and source are credited

Protocol status: Working We use this protocol and it's working

Created: Apr 09, 2019

Last Modified: Apr 10, 2019

PROTOCOL integer ID: 22199

\section{(3) In Vitro Transcription for dgRNA V.1}

Amy Lyden ${ }^{1}$, Emily Crawford ${ }^{2}$, Jenai Quan ${ }^{1}$, Saharai Caldera $^{2}$, Lara Pesce-Ares ${ }^{1}$

${ }^{1}$ CZ Biohub; ${ }^{2}$ CZ Biohub, UCSF<smiles>C1CCCCCC1</smiles>

Amy Lyden

\section{ABSTRACT}

For FLASH, DASH, and other CRISPR-cas9 protocols, we use T7 to transcribe our crRNA and tracrRNA to make dgRNA for cas9. It is more time-, labor-, and costeffective to make dgRNAs instead of sgRNAs for large guide RNA libraries such as those used in DASH or FLASH. The two components of the dual guides are the crRNA (containing your variable $20 \mathrm{nt}$ target plus a $22 \mathrm{nt}$ constant region) and the tracrRNA (a $72 \mathrm{nt}$ constant region).

\section{GUIDELINES}

Work in an RNAse free space! If possible, work inside a PCR workstation/hood, in a pre-PCR environment.

\section{MATERIALS}

\section{MATERIALS}

88 Thermocycler Contributed by users

88 Ethanol $100 \%$ Contributed by users

88 NanoDrop spectrophotometer Thermo Fisher Scientific Catalog \#ND-1000

88 Nuclease-free water Ambion Catalog \#AM9932

88 Qubit RNA HS Assay Kit Thermo Fisher Scientific Catalog \#Q32852

88 SPRI beads (homemade) or Ampure XP beads Contributed by users

88 crRNA template (60nt) IDT

88 tracrRNA template (90nt) IDT

10X T7 Buffer (400 mM Tris pH 7.9 - 200 mM MgCl2 - 50 mM DTT - 20 mM

spermidine (Sigma 85558)) store at $-80 \mathrm{C}$ Contributed by users

88 T7 Enzyme $(10 \mathrm{mg} / \mathrm{mL})$ Contributed by users

88 NTP Set 100 mM Solution Thermo Fisher Scientific Catalog \#R0481

8 Magnetic Tube Rack for $1.5 \mathrm{~mL}$ or $15 \mathrm{~mL}$ tubes Contributed by users Catalog \#12321D

$8 \&$ T7 transcription primer (18nt) IDT

STEP MATERIALS 
Keywords: DASH, FLASH, cas9, dgRNA, IVT, transcription
88 crRNA template (60nt) IDT

88 T7 transcription primer (18nt) IDT

88 tracrRNA template (90nt) IDT

88 T7 transcription primer (18nt) IDT

88 NanoDrop spectrophotometer Thermo Fisher Scientific Catalog \#ND-1000

88 NTP Set 100 mM Solution Thermo Fisher Scientific Catalog \#R0481

10X T7 Buffer (400 mM Tris pH 7.9 - 200 mM MgCl2 - 50 mM DTT - 20 mM

spermidine (Sigma 85558)) store at $-80 \mathrm{C}$ Contributed by users

88 T7 Enzyme $(10 \mathrm{mg} / \mathrm{mL})$ Contributed by users

$\& 8$ tracrRNA template (90nt) IDT

88 Nuclease-free water Ambion Catalog \#AM9932

88 crRNA template (60nt) IDT

88 Nuclease-free water Ambion Catalog \#AM9932

88 SPRI beads (homemade) or Ampure XP beads Contributed by users

88 Ethanol $100 \%$ Contributed by users

88 Qubit RNA HS Assay Kit Thermo Fisher Scientific Catalog \#Q32852

88 Agilent Small RNA Bioanalyzer kit Contributed by users Catalog \#5067-154

- NTP quality varies from one vendor to another. We have had consistent success with Thermo cat \# r0481 and Life Tech AM81110G, -20G, -30G, and-40G, used at a final concentration of $1 \mathrm{mM}$ each

- We purify our own T7, and experiments should be optimized for each batch of T7, or for a commercial T7.

\section{BEFORE START INSTRUCTIONS}

\section{Designing the crRNA(s): (contains your target sequence)}

$\square$ S. pyogenes cas9 requires a 20-nt target directly 5' to a PAM motif "NGG" (where $\mathrm{N}$ is any nucleotide). The NGG is not present in the guide RNA itself. So when choosing a target you are looking for a sequence that matches the following pattern (and don't forget that you can target either strand):

\section{5'----NNNNNNNNNNNNNNNNNNNNNGG----3'}

or 5'----CCNNNNNNNNNNNNNNNNNNNNN----3'

where the $20 \mathrm{Ns}$ in bold are your target site. Cas 9 will cut between the $17^{\text {th }}$ and $18^{\text {th }}$ nt of the target, yielding the following products:

5'----NNNNNNNNNNNNNNNNN3' 5'NNNNGG----3'

or 5'----CCNNNN3' 5'NNNNNNNNNNNNNNNNN----5' 
The sequence of each crRNA should be as follows, with the Ns replaced by your 20 nt target:

TAATACGACTCACTATAGNNNNNNNNNNNNNNNNNNNNGTTTTAGAGCTATGCTG TTTTG

The underlined portion is the T7 transcription site. T7 only requires its own $18 \mathrm{nt}$ binding site to be double-stranded; the rest of the template can be single stranded. Thus the template can be constructed by purchasing two oligos from IDT: the reverse complement of the $60 \mathrm{nt}$ sequence listed above, plus an $18 \mathrm{nt}$ oligo to make the T7 site double stranded:

60mer reverse complement:

CAAAACAGCATAGCTCTAAAACNNNNNNNNNNNNNNNNNNNNCTATAGTG AGTCGTATTA

18mer T7:

TAATACGACTCACTATAG

The tracrRNA: (constant for all dgRNA)

The sequence of the tracrRNA template should be as follows:

TAATACGACTCACTATAGGACAGCATAGCAAGTTAAAATAAGGCTAGTCCGTTATCA ACTTGAAAAAGTGGCACCGAGTCGGTGCTTTTT

Just as with the crRNA, only the T7 binding site needs to be double stranded, so the following two oligos can be purchased from IDT:

90mer reverse complement:

AAAAAGCACCGACTCGGTGCCACTTTTTCAAGTTGATAACGGACTAGCCTTA TTTTAACTTGCTATGCTGTCCTATAGTGAGTCGTATTA

18mer T7:

TAATACGACTCACTATAG

\section{Annealing T7 to crRNA and tracrRNA template}

1 Pool your crRNA in equimolar amounts. Usually, we order 96-well plates of 96 crRNA templates from IDT, with the oligos diluted in water at a concentration of $10 \mu \mathrm{M}$ 


\section{$\& 8$ crRNA template (60nt) IDT}

2 Add an equimolar amount of T7 to your crRNA pool. For example, reconstitute T7 to $10 \mu \mathrm{M}$, and pool $500 \mu \mathrm{L}$ of your $10 \mu \mathrm{M}$ crRNA pool with $500 \mu \mathrm{L}$ of your T7 at $10 \mu \mathrm{M}$.

\section{T7 transcription primer (18nt) IDT}

3 Add an equimolar amount of T7 to your tracr RNA. For example, if you have reconstituted your tracrRNA to $100 \mu \mathrm{M}$, pool $500 \mu \mathrm{L}$ of your tracrRNA at $100 \mu \mathrm{M}$ to $500 \mu \mathrm{L}$ of your T7 at $100 \mu \mathrm{M}$.

\section{tracrRNA template (90nt) IDT}

88 T7 transcription primer (18nt) IDT

4 Anneal tracrRNA + T7 and crRNA + T7 by heating to $8^{\circ} 95^{\circ} \mathrm{C}$ on a heat block or thermocycler for 00:02:00 and allowing them to cool to room temperature slowly on the bench

\section{Prepare for IVT reaction}

$5 \quad$ Nanodrop or Qubit your tracrRNA and crRNA using ssDNA setting or kit.

88 NanoDrop spectrophotometer Thermo Fisher Scientific Catalog \#ND-1000

6 Dilute your tracrRNA with T7 annealed to $800 \mathrm{ng} / \mu \mathrm{L}$ with water. Dilute your crRNA with T7 to $40 \mathrm{ng} / \mu \mathrm{L}$ with water.

Note

Can store these as aliquots at $8^{\circ}-20^{\circ} \mathrm{C}$ 


\section{In Vitro Transcription}

$7 \quad$ Make all reagents are at room temperature. Prepare the reaction mixtures below in the order specified. DO NOT prepare the reaction on ice, as some components are prone to precipitation.

8 Mix NTPs together in equimolar amounts to have enough for the following reactions. For example, mix $200 \mu \mathrm{L}$ A NTP at $100 \mathrm{mM}, 200 \mu \mathrm{L} C$ NTP at $100 \mathrm{mM}, 200 \mu \mathrm{L}$ G NTP at $100 \mathrm{mM}$, and $200 \mu \mathrm{L} U \mathrm{NTP}$ at $100 \mathrm{mM}$ for a final solution of $25 \mathrm{mM}$ each.

\section{NTP Set 100 mM Solution Thermo Fisher Scientific Catalog \#R0481}

9 Prepare a small amount of 1 X T7 buffer $(200 \mu \mathrm{L}$ for the reaction below). Dilute your T7 enzyme to $100 \mu \mathrm{g} / \mathrm{mL}$ in $1 \mathrm{X}$ T7 buffer. 85558)) store at $-80 \mathrm{C}$ Contributed by users

88 T7 Enzyme $(10 \mathrm{mg} / \mathrm{mL})$ Contributed by users

10

Prepare crRNA mixture by adding the following reagents to a $1.5 \mathrm{~mL}$ tube in order.

\section{crRNA template (60nt) IDT}

88 Nuclease-free water Ambion Catalog \#AM9932

\begin{tabular}{|l|l|}
\hline Volume & crRNA \\
\hline $1 \mathrm{X}$ & \\
\hline $380 \mu \mathrm{L}$ & RNAse-free water \\
\hline $120 \mu \mathrm{L}$ & $10 X$ T7 buffer* \\
\hline $300 \mu \mathrm{L}$ & NTPs 25mM each \\
\hline
\end{tabular}




\begin{tabular}{|l|l|}
\hline $100 \mu \mathrm{L}$ & $\begin{array}{l}\text { T7 enzyme (1:100 } \\
\text { diluted in 1X T7 } \\
\text { buffer, final conc: } \\
100 \mu \mathrm{g} / \mathrm{mL})\end{array}$ \\
\hline $100 \mu \mathrm{L} / 4 \mu \mathrm{g}$ & $\begin{array}{l}\text { crRNA template with } \\
\text { T7 annealed, } \\
40 \mathrm{ng} / \mu \mathrm{L}\end{array}$ \\
\hline $1 \mathrm{~mL}$ & TOTAL \\
\hline
\end{tabular}

*Experiments indicated that treating the $10 x \mathrm{~T} 7$ buffer like $8.3 \mathrm{x}$ improved yields

11 Prepare tracrRNA mixture by adding the following reagents to a $1.5 \mathrm{~mL}$ tube in order.

\section{8 tracrRNA template (90nt) IDT}

88 Nuclease-free water Ambion Catalog \#AM9932

\begin{tabular}{|l|l|}
\hline Volume & tracrRNA \\
\hline $1 \mathrm{X}$ & RNAse-free water \\
\hline $470 \mu \mathrm{L}$ & $10 X \mathrm{T7}$ buffer* \\
\hline $120 \mu \mathrm{L}$ & NTPs $25 \mathrm{mM}$ each \\
\hline $300 \mu \mathrm{L}$ & $\begin{array}{l}\text { T7 enzyme }(1: 100 \\
\text { diluted in } 1 \text { T T7 } \\
\text { buffer, final conc: } \\
100 \mu \mathrm{mL})\end{array}$ \\
\hline $100 \mu \mathrm{L}$ & $\begin{array}{l}\text { tracrRNA template } \\
\text { with T7 annealed, } \\
800 \mathrm{ng} / \mu \mathrm{L}\end{array}$ \\
\hline $10 \mu \mathrm{L} / 8 \mu \mathrm{g}$ & \begin{tabular}{l} 
TOTAL \\
\hline $1 \mathrm{~mL}$
\end{tabular} \\
\hline
\end{tabular}

*Experiments indicated that treating the $10 \mathrm{x}$ T7 buffer like $8.3 \mathrm{x}$ improved yields 
12 Incubate at $8^{\circ} 37^{\circ} \mathrm{C}$ for $02: 00: 00$ and proceed immediately to purification.

\section{RNA Purification with SPRI beads}

13 Use homemade SPRI beads or Ampure beads to purify gRNAs after transcription.

SPRI beads (homemade) or Ampure XP beads Contributed by users

\section{Note}

Note: Alternatively, RNA may be purified using a Zymo RNA Clean \& Concentrator-5 or -25 Kit (Zymo Research R1015)

14 Equilibrate SPRI beads to room temperature.

15 For every $200 \mu \mathrm{L}$ of IVT reaction, add $300 \mu \mathrm{L}$ of $100 \%$ ethanol. The solution should turn a cloudy white (precipitation of RNA) upon addition of ethanol. This step helps the short RNAs bind to the SPRI beads. This can be done in a $15 \mathrm{~mL}$ tube or several $1.5 \mathrm{~mL}$ tubes.

\section{Ethanol $100 \%$ Contributed by users}

16 Add $500 \mu \mathrm{L}$ of SPRI beads to the solution of IVT reaction and ethanol and mix well by inverting or pipetting with a P1000.

17 Incubate at room temperature for 00:05:00

18 Divide this mixture up into an appropriate number of 1.5mL Lo-Bind Eppendorf tubes and place on a $1.5 \mathrm{~mL}$ magnetic separation rack OR use a $15 \mathrm{~mL}$ magnetic tube rack. 
19 Wait 00:05:00 to allow the beads to separate if using a 1.5mL rack OR 00:15:00 if using a $15 \mathrm{~mL}$ rack.

20 Remove and discard the supernatant.

21 Rinse the beads with $1 \mathrm{~mL}$ of $80 \%$ ethanol if using a $1.5 \mathrm{~mL}$ tube or $\sim 10 \mathrm{~mL}$ of $80 \%$ ethanol if using the $15 \mathrm{~mL}$ tube. It is not necessary to resuspend the beads.

22 Wait 00:01:00 then remove and discard the ethanol.

23 Repeat the wash step as described above. (Add the same amount of $80 \%$ ethanol, wait 00:01:00, then discard the ethanol.)

24 Remove residual ethanol that collects at the bottom of the tube by using a P200 or P20.

25 Air dry the beads for $00: 05: 00$ in a $1.5 \mathrm{~mL}$ tube or $00: 15: 00$ in a $15 \mathrm{~mL}$ tube, or until the beads lose their glossy appearance. Sufficiently dry beads will appear matte. Be careful not to let the beads get too dry (appearing cracked or dusty).

26 Elute the RNA by resuspending the beads with an appropriate amount of nuclease-free $\mathrm{H} 2 \mathrm{O}$ depending on the desired volume and concentration. For DASH guides which need to be at a final 
concentration of $40 \mu \mathrm{M}$, lower is better $(80 \mu \mathrm{L}$ per $1 \mathrm{X}$ reaction). For FLASH guides which need to be at a final concentration of $4 \mu \mathrm{M}$, a higher volume can be used.

27 Allow the RNA to elute off the beads by incubating at room temperature for 00:05:00

28 If necessary, pulse-spin the tubes to collect any liquid along the sides of the tubes.

29 Place the tubes on the magnetic rack and allow them to separate until water is clear. This will take $2-5$ minutes for a $1.5 \mathrm{~mL}$ tube, $5-10$ minutes for a $15 \mathrm{~mL}$ tube.

30 Collect the eluted RNA, being careful not to take up beads. (Eg. If eluted in $80 u \mathrm{~L}$, collect $75 \mu \mathrm{L}$ ).

\section{Quantify, anneal and aliquot dgRNA}

31 Using the HS RNA Qubit kit, quantify $1 \mu \mathrm{L}$ of the eluted tracrRNA and $1 \mu \mathrm{L}$ of the eluted crRNA. Follow standard HS RNA Qubit protocol.

\section{Qubit RNA HS Assay Kit Thermo Fisher Scientific Catalog \#Q32852}

32 crRNA: For DASH, dilute the stock crRNA to $1100 \mathrm{ng} / \mu \mathrm{L}$. This is equivalent to $80 \mu \mathrm{M}$. For FLASH or other lower concentration needs, dilute to $110 \mathrm{ng} / \mu \mathrm{L}$ or $8 \mu \mathrm{M}$

33 tracrRNA: For DASH, dilute the stock crRNA to $1900 \mathrm{ng} / \mu \mathrm{L}$. This is equivalent to $80 \mu \mathrm{M}$. For FLASH or other lower concentration needs, dilute to $190 \mathrm{ng} / \mu \mathrm{L}$ or $8 \mu \mathrm{M}$

34 If you want to check purity, run your 1:100 dilutions on a small RNA chip on the bioanalyzer 
immediately after denaturing them by heating to $8^{\circ} 95^{\circ} \mathrm{C}$ for $00: 03: 00$. The crRNA and tracrRNA are $42 \mathrm{nt}$ and $72 \mathrm{nt}$ long, respectively.

\section{Agilent Small RNA Bioanalyzer kit Contributed by users Catalog \#5067-1548}

35 To form the dgRNA complex, mix together equilmolar amounts of crRNA and tracrRNA (equal volumes of the $80 \mu \mathrm{M}$ crRNA stock and the $80 \mu \mathrm{M}$ tracrRNA stock), heat to $8^{\circ} 95^{\circ} \mathrm{C}$ for 00:00:30 and then cool slowly on the bench.

36 Store dgRNA at $8^{\circ}-80^{\circ} \mathrm{C}$ in small aliquots in order to avoid freeze-thaws. If there is crRNA or tracrRNA left over, freeze them separately -- the dgRNA complex can be formed in the same way $8^{\circ} 95^{\circ} \mathrm{C}$ for $00: 00: 30$ immediately prior to complexing with Cas 9. 